\title{
BMJ Open Studying Accompaniment model Feasibility and Effectiveness (SAFE) Study: study protocol for a prospective observational cohort study of the effectiveness of self-managed medication abortion
}

\author{
Heidi Moseson (1) , ${ }^{1}$ Brianna Keefe-Oates, ${ }^{2}$ Ruvani T Jayaweera, ${ }^{1}$ Sofia Filippa, ${ }^{3}$ \\ Relebohile Motana, ${ }^{4}$ Chiara Bercu, ${ }^{1}$ Ijeoma Egwuatu, ${ }^{5}$ Belen Grosso, ${ }^{6}$ \\ Ika Ayu Kristianingrum, ${ }^{7}$ Sybil Nmezi, ${ }^{5}$ Ruth Zurbriggen, ${ }^{6}$ Emmeline Friedman, ${ }^{8}$ \\ Caitlin Gerdts ${ }^{1}$
}

To cite: Moseson H, KeefeOates B, Jayaweera RT, et al. Studying Accompaniment model Feasibility and Effectiveness (SAFE) Study: study protocol for a prospective observational cohort study of the effectiveness of self-managed medication abortion. BMJ Open 2020;10:e036800. doi:10.1136/ bmjopen-2020-036800

- Prepublication history and additional material for this paper are available online. To view these files, please visit the journal online (http://dx.doi. org/10.1136/bmjopen-2020036800).

Received 06 January 2020 Revised 29 September 2020 Accepted 19 October 2020

D) Check for updates

(c) Author(s) (or their employer(s)) 2020. Re-use permitted under CC BY-NC. No commercial re-use. See rights and permissions. Published by BMJ.

For numbered affiliations see end of article.

Correspondence to Dr Heidi Moseson; hmoseson@ibisreproductive health.org

\section{ABSTRACT}

Introduction A range of barriers deter or prevent people from accessing facility-based abortion care. As a result, people are obtaining and using abortifacient medications to end their pregnancies outside of the formal healthcare system, without clinical supervision. One model of self-managed abortion has come to be known as the 'accompaniment' model, in which grassroots organisations provide pregnant people with evidence-based counselling and support through the medication abortion process. Data are needed to understand the safety and effectiveness of this increasingly common model of abortion care.

Methods and analysis This is a large, prospective, observational study in Argentina and Nigeria. All people who contact one of two accompaniment groups seeking information for their own self-managed medication abortion, are ages 13 years and older, have no contraindications for medication abortion, are within the gestational range supported by the group (up to 12 weeks' gestation for the primary outcome) and are willing to be contacted for follow-up will be recruited. Participants will respond to an interviewer-administered baseline survey at enrolment, and 1-4 additional surveys over 6 weeks to ascertain whether they obtain medications for abortion, dosing and route of administration of medications, physical and emotional experience of medication abortion selfmanagement, and effectiveness and safety outcomes. Analyses will include estimates of the primary outcome: the proportion of participants that report a complete abortion without surgical intervention at last recorded follow-up; as well as secondary outcomes including a pseudo-experimental test of non-inferiority of the effectiveness of self-managed medication abortion as compared with clinical medication abortion.

Ethics and dissemination We describe the ethical considerations and protections for this study, as well the creation of a study-specific Data Monitoring and Oversight Committee. We describe dissemination plans to ensure that study results are shared widely with all

\section{Strengths and limitations of this study}

- This study will provide new information on the safety and effectiveness of self-managed medication abortion with support from trained volunteers, outside of the formal healthcare system.

- The primary outcome will be the proportion of people who report a complete abortion without surgical intervention after self-use of misoprostol, alone or in combination with mifepristone, outside of the healthcare setting.

- Secondary outcomes will include an evaluation of whether self-managed medication abortion effectiveness is non-inferior to medication abortion effectiveness in a clinical setting, as well as information on medication dosing and timing, duration of the abortion process, details of the physical experience, including pain management, and experiences of healthcare seeking.

- Findings from this study could shift the global conversation around de-medicalised abortion, and inform revisions to global task-shifting guidelines for who can be a safe abortion provider.

- A limitation of this study is the inability to recruit a formal control group, due to legal restrictions on abortion in the study settings; thus, we rely on historical controls for the pseudo-experimental noninferiority analysis.

relevant audiences, particularly researchers, advocates, policymakers and clinicians.

Trial registration number ISRCTN95769543.

\section{INTRODUCTION}

Access to safe and effective methods of abortion is an essential component of sexual and reproductive healthcare, and necessary for 
the realisation of the human right to bodily autonomy. However, legal restrictions, lack of willing and trained providers, high costs, long wait times for services and abortion stigma all serve as barriers to accessing safe abortions within the formal healthcare system. ${ }^{1-4}$ People around the world are increasingly obtaining and using the abortifacient medications mifepristone and/or misoprostol to end their pregnancies outside of the formal healthcare system, without clinical supervision $\left(\mathrm{eg},{ }^{5-10}\right)$. Some people use the medications on their own with information from the Internet or friends, while some seek guidance from pharmacists, safe abortion hotlines and websites, and accompaniment groups. ${ }^{9}$ We describe the use of medications to induce abortion outside of the formal healthcare system without clinical supervision as self-managed abortion.

An emerging body of evidence suggests that nearly half of abortions worldwide are self-managed, ${ }^{11}$ and up to $70 \%$ or $80 \%$ in some settings. ${ }^{12}$ People self-manage their abortions for many reasons, ranging from a preference for the privacy inherent in the model to using a method of last resort when facility-based care is inaccessible. ${ }^{9}$ One model of information and support for self-managed abortion has come to be known as the 'accompaniment' model, in which grassroots organisations provide people with evidence-based counselling and support through the medication abortion process outside of the formal healthcare system. ${ }^{13}{ }^{14}$ There is growing awareness of these accompaniment models and increasing recognition of the role of the individual in safely managing their own abortion. In 2015, the World Health Organization (WHO) released guidelines that outlined taskshifted roles for health workers in the provision of safe abortion. ${ }^{15}$ For the first time, these guidelines included the pregnant person as an actor in their own abortion process, and acknowledged that the experience of selfmanagement of abortion can be empowering and could lead to a more optimal use of scarce health resources. ${ }^{15}$

Decades of evidence have demonstrated that medication abortion with misoprostol alone or in combination with mifepristone is an effective and safe method of abortion when administered in a clinic setting. ${ }^{16-20}$ Yet, concerns remain that people may not be able to use these medications to safely and effectively induce abortion outside of the formal healthcare system without clinical supervision, either due to inaccurate self-assessment of gestational age, inability to follow dosing instructions, or concerns about access to care in the event of a complication or adverse event. ${ }^{21}$ We hypothesise, however, that with counselling from accompaniment groups, people can safely and effectively self-manage abortion with medication. This hypothesis is informed by a strong and growing body of evidence on the effectiveness and safety of self-managed medication abortion. ${ }^{568922{ }^{23}}$ Existing research has analysed records of self-managed medication abortion from online telemedicine groups, as well as community health workers, and more. ${ }^{568}$ However, some limitations of existing data, such as a reliance on retrospective records not collected for research purposes, and a scarcity of research on accompaniment models specifically, hinder the field's understanding of the effectiveness and safety of this particular model of abortion care: self-managed medication abortion with accompaniment group support.

Well-designed, rigorously collected research that evaluates the effectiveness and safety of self-managed medication abortion via accompaniment models is needed to understand the experiences of people who use this increasingly common model of abortion care, and to decide if and how to support new non-clinical models of abortion care in a range of legal settings.

\section{Objectives}

In this manuscript, we present the protocol for the Studying Accompaniment model Feasibility and Effectiveness Study (the SAFE Study). The main objective of the SAFE Study is to estimate the effectiveness of both mifepristone with misoprostol, and misoprostol-only, regimens when used to terminate a pregnancy outside of the formal healthcare system, without clinical supervision but with support from accompaniment groups. A secondary objective is to compare the effectiveness of self-managed medication abortion with accompaniment group support to the effectiveness of medication abortion when administered in the clinical setting (a pseudo-experimental noninferiority analysis). Beyond these core aims, additional objectives include describing the physical experience of self-managed abortion, including incidence and severity of side effects and potential signs of complications, interactions with the formal healthcare system, including surgical and other interventions, emotions throughout the self-managed abortion process, and more.

\section{METHODS AND ANALYSIS}

The SAFE Study is a large, prospective observational cohort study in two countries: Argentina and Nigeria. These countries were selected for the study based on (1) the existence of accompaniment groups that serve a sufficiently high number of clients required to reach the target sample size, (2) geographical representation, (3) organisational interest in the research question and (4) diversity in availability of abortion medications outside of the formal healthcare setting. In both countries, abortion is allowed only on the grounds of preserving health or saving the pregnant person's life; as a result, abortion is not widely available within the formal healthcare setting. ${ }^{24}$

The accompaniment groups selected as recruitment partners for this study vary in their modes of operation, but both provide step-by-step guidance for how to use medication to safely induce abortion based on the WHO protocols (table 1) ${ }^{25}{ }^{26}$ Counsellors are trained to provide empathetic, non-judgemental, gestational agespecific, and evidence-based information to individuals who contact these groups in need of information and support in ending their pregnancy with medications. 
Table 1 Medication abortion protocols

Mifepristone+misoprostol for pregnancies up to 84 days

- Swallow 1 tablet of mifepristone $(200 \mathrm{mg})$ with a glass of water

- After 36-48hours, put 4 pills of misoprostol $(800 \mu \mathrm{g})$ under the tongue (sublingual) and let them dissolve for $30 \mathrm{~min}$, keep swallowing saliva until the pills dissolve.

If after 3 hours there are no signs of reaction, side effects or expulsion, put 2 additional misoprostol pills $(400 \mu \mathrm{g})$ under the tongue, and let them dissolve for $30 \mathrm{~min}$.
Misoprostol alone for pregnancies up to 84 days

- Put 4 pills $(800 \mu \mathrm{g})$ under the tongue (sublingual) and let them dissolve for $30 \mathrm{~min}$, keep swallowing saliva until the pills dissolve. Wait for 3 hours.

- After 3 hours, put the second dose of 2-4 pills $(400-800 \mu \mathrm{g})$ under the tongue and let them dissolve for $30 \mathrm{~min}$, keep swallowing saliva until the pills dissolve. Wait for 3 hours.

- After 3 hours, put a third dose of 2-4 pills (400-800 $\mu \mathrm{g})$ under the tongue and let them dissolve for $30 \mathrm{~min}$, keep swallowing saliva until the pills dissolve.

Continue with 2-4 misoprostol pills under the tongue every 3 hours until expulsion occurs.
In addition to information about medication abortion protocols, counsellors may also provide information on how to obtain medications, how the drugs function, how to manage pain, how to recognise complication signs, how to prepare for potential interactions with medical personnel in case of emergency-treatment seeking, how to confirm abortion completion, what to expect after the abortion and prevention of future unwanted pregnancy.

\section{Patient and public involvement}

A research consortium that includes researchers, activists and accompaniment providers collaboratively developed the study protocol detailed below to ensure that it reflects the priorities, experiences and preferences of people who self-manage abortions with medication. Study investigators invited individual consortium members to participate based on their expertise in self-managed medication abortion and accompaniment models in a range of legal and cultural settings, to ensure the design of a study that reflected the lived experiences of people who self-manage, the accompaniers who support them, and contexts similar to those in which the study will take place. Consortium members also played an active role in ensuring that study instruments would collect data that could be useful for people involved with self-managed medication abortion around the world, and minimally burdensome. Each consortium member drew on their knowledge of people's priorities, experiences and preferences to finalise the research question and outcome measures. Individual 'patients' participated in cognitive interviews and pilot testing to ensure that study questions and procedures were acceptable to and relevant for those pursuing this model of abortion care. The recruitment sites recruited 227 participants during a 60-day pilot study between April and June 2019 to assess the feasibility and acceptability of the study procedures, and conducted postpilot in-depth interviews with participants to understand the participant experience. ${ }^{27}$ The research consortium discussed pilot study results and experiences in-depth; and as a result, the consortium proposed modifications in eligibility criteria and timing of questions for the full study as detailed below, to minimise burden to participants and improve data quality.

In addition to the research consortium that designed the study, study investigators also invited four individuals to serve on a study-specific Data Monitoring and Oversight Committee (DMOC) to provide expert guidance to the SAFE Study research consortium throughout the research process. The overall role of the DMOC is to assist the SAFE Study investigators in protecting the interests of study participants and in preserving the integrity and credibility of the study. The four DMOC members have expertise in epidemiology, survey methods, statistics, participant advocacy and self-managed medication abortion, as well as personal connections to the countries included in this study, and have reviewed the pilot study protocol, pilot study results and full study protocol. They will also review interim study results to evaluate participant safety and other protections.

\section{Study participants}

Each person that contacts one of the organisations during the study period will be screened for eligibility by the organisation staff. Eligible participants will be those who: contacted the accompaniment group seeking information about induced abortion for their own pregnancy; are at least 13 years of age; are able to give informed consent; are able to speak one of the local languages; meet accompaniment group eligibility criteria for starting a medication abortion process (ie, no contraindications to medication abortion, within a gestational age range that the accompaniment group supports-up to 24 weeks in Argentina in limited circumstances, and up to 15 weeks in Nigeria); and are starting a new medication abortion process. Counselors ask each caller to provide an estimated gestational age for the pregnancy and to indicate whether this gestational age estimate has been confirmed by ultrasound. If a caller has not had an ultrasound, counselors enter the caller's self-reported date of last menstrual period into a calendar-based gestational age calculator to confirm estimated gestational age. Individuals will be excluded if they are beyond the gestational 
age range supported by the accompaniment group, experiencing ongoing symptoms (bleeding, cramping) from a prior attempt at induced abortion or that could indicate a miscarriage; have a known ectopic pregnancy or symptoms of an ectopic pregnancy; do not want to share their contact information with study staff; do not want to be contacted again by the accompaniment group or by study staff; are not willing to comply with study procedures; or cannot access a phone and private location to answer questions during follow-up in the approximately 3-6 weeks of follow-up.

Across all sites, screening and invitation to participate will take place after each person has received the initial counselling from the organisation, which includes details on evidence-based protocols for medication abortion. Screening and invitation will take place either over the phone or in person, depending on the organisation's model of providing counselling. Accompaniment group counsellors will assess client eligibility over the course of the counselling process; if the person is eligible, they will invite them to participate, and if they express interest, they will proceed through an informed consent process. Verbal consent will be obtained. Special emphasis will be placed on potential participants less than 18 years of age, for whom counsellors will be trained to describe study participation in familiar terms, to ensure that any young person enrolled understands the risks and benefits, and is actively willing to participate, rather than merely failing to object. For people that consent to participate, the counsellor will sign and date a paper informed consent form as record of informed consent, will record a participantapproved identifier for that person, assign a unique study identification (ID) number, collect detailed contact information for follow-up and notify the study coordinators at each site of the new enrollee to schedule subsequent follow-ups.

\section{Sample size}

To assess the sample size needed to estimate the overall effectiveness of self-managed medication abortion through 84 days' ( 12 weeks, 0 days) gestation, across medication abortion regimens and accompaniment groups, we looked to clinical effectiveness estimates of medication abortion from clinical trials. Existing data suggest that we might expect to see an effectiveness of $93 \%$ for mifepristone and misoprostol users through 84 days' gestation and $80 \%$ effectiveness for users of misoprostol alone through 84 days' gestation. ${ }^{1618-2028} 29$ Under these assumptions, we will need to recruit a minimum of 213 subjects in the mifepristone and misoprostol setting (Argentina), and approximately 419 misoprostol-only users and 77 mifepristone and misoprostol users (Nigeria), based on observed proportions of callers in each site using the combined regimen, versus misoprostol alone. Thus, we will recruit and prospectively follow a minimum of 709 accompaniment model callers across the two countries.

However, to account for expected loss to follow-up of $10 \%$ based on a 60 -day pilot study conducted at both sites, and to be able to evaluate secondary outcomes with greater statistical power, we have increased our target sample size beyond the minimum numbers. Thus, our target sample size for each site is 400 in Argentina and 600 in Nigeria. This sample size will allow us to (1) assess our primary outcome (the proportion with a complete abortion without surgical evacuation) within a $5 \%$ margin, $90 \%$ power and an alpha of $5 \%$; while also allowing us to evaluate our secondary outcome, (2) the pseudoexperimental non-inferiority test, to assess whether selfmanaged medication abortion with accompaniment support is no more than $5 \%$ less effective than the clinical setting for each regimen, with $80 \%$ power, an alpha of $5 \%$ and assuming no correlation within counsellors (based on pilot study results by site).

The study, like other medication abortion studies,${ }^{30}$ will not be powered to detect a difference in safety outcomes between regimens as major adverse events attributable to medication abortion are extremely rare, but we will document the occurrence of these rare events. ${ }^{31}$ Participants beyond 84 days' gestation will be eligible to participate to gather needed data on self-managed medication abortion outcomes within these understudied gestational ranges, but will not count toward the minimum sample size or be included in the evaluation of the primary effectiveness outcome.

\section{Data collection and data management}

At each site, study coordinators will be responsible for monitoring enrolment and conducting follow-up calls. All enrolled participants will be asked a set of questions immediately after enrolment in the study; additional baseline information will be extracted from the caller's counselling record by the accompaniment counsellor or study coordinator. Baseline questions include sociodemographic characteristics, reproductive history, gestational age and preferred mode of contact (phone call, short message service or messaging application). All study instruments are included in online supplemental file 1 . In Argentina, in addition to the baseline information collected at all three sites, a sub-sample of $25 \%$ of participants will be asked to take a pregnancy test at baseline to confirm that participants are pregnant.

At enrolment, the counsellor will record the estimated date that the participant plans to start their medication abortion process. Approximately 1 week after the estimated/confirmed date of starting the medication abortion process, the study coordinator will follow up with the participant via their preferred mode of contact. At this first follow-up, the study coordinator will record the following outcomes as reported by the participant: (1) if and when the participant successfully obtained abortion medications; (2) if and when the participant has taken the medication (time, date and route for each dose); (3) if and when the participant completed the abortion, and how the completion was confirmed; (4) if, when, why and what type of healthcare was sought at a healthcare facility; 
and (5) details on the symptoms and side effects experienced during the medication abortion process.

For participants at enrolment who do not know if or when they plan to obtain or take the pills, or who do not remain in touch with a counsellor about their plans, the study coordinator will follow up 2 weeks after the date of enrolment to conduct the first follow-up. At this first follow-up, if the participant reports that they have not obtained pills and do not plan to continue with the medication abortion, or that they have obtained the pills but do not plan to use them, the study coordinator will not contact them again, and their outcome will be recorded as 'Decided to continue their pregnancy', 'Miscarriage', 'Decided to obtain a surgical abortion' or another outcome, as appropriate. If the participant reports that they have the pills and have taken them (or plan to), follow-up will be reset to 7 days following the date they report taking (or planning to take) the pills, and will proceed as outlined above.

For all participants that report taking the medications, the study coordinator will conduct a second follow-up approximately 3 weeks following the first dose of medication (2 weeks after the first follow-up), to assess the primary outcome (self-report of complete abortion) and any subsequent complications, as well as secondary outcomes. For participants whose abortion outcome cannot be ascertained by the second follow-up, the study coordinator will reach out for a third follow-up 1 week later (4weeks from first dose of medication) to document primary and secondary outcomes. If the participant's outcome still cannot be ascertained at the third follow-up, the study coordinator will contact them one final time 2 weeks later (6weeks after taking the pills). For any follow-up point, if after four attempts the study coordinator has still not been able to contact the participant, that participant will have missing data for that time point. Contact will be attempted again for all participants for any following surveys, even if a participant was 'missing' for a prior follow-up. When possible, the study coordinators will input any missing data based on data available in the counselling record for a particular participant. All participants will be compensated for their time in a form and amount that is deemed appropriate for each setting, approximately US\$10-25 total in telephone credit or mobile money voucher over the course of the study. Figure 1 displays a representation of the study procedures.

Study coordinators will record all survey responses first on paper forms, and then will manually enter data from all paper records into Qualtrics (Qualtrics, Provo, Utah, USA). A unique study ID number for each participant will link data across follow-ups by participant. All physical study data (screening forms, counselor-signed informed consent forms and completed paper surveys) will be stored in locked filing cabinets and only the local study coordinators will have the key. Once transferred to an encrypted, password-protected electronic file, all paper forms that link participant alias to study ID number will be destroyed. Electronic survey data in Qualtrics will be stored in Qualtrics' password-protected and encrypted cloud storage.

Data will be entered electronically to allow for ongoing monitoring of data quality, and to utilise electronic checks to promote complete data entry. Quantitative data completeness will be assessed first by the study coordinators at each follow-up, reviewing data from the prior follow-up to assess completeness, and on the aggregate level by the team leads on a monthly basis. Interim review of the paper surveys, consent forms, and data entry and storage processes will further ensure the quality of data collected, and will allow for opportunities to swiftly resolve any gaps or challenges that arise during data collection.

\section{Data analysis}

The primary outcome of this study is the proportion of participants who report a complete abortion without surgical evacuation at last recorded study follow-up. This will be determined by the proportion of participants who report 'yes' to the question 'Do you feel that your abortion process is complete?' at their last recorded study follow-up, and who do not report any surgical intervention. Research suggests that people are able to self-assess medication abortion completion accurately. ${ }^{32}$ For the primary outcome, consistent with other studies of medication abortion effectiveness, ${ }^{28}$ we will calculate the proportion with a complete abortion without surgical intervention among all participants who reported taking medications and have a known abortion outcome. Sensitivity analyses will evaluate this proportion among all participants who reported taking medications (were exposed), and will conservatively assume that those with missing outcome data had a failed abortion.

Exploratory subgroup analyses will evaluate this proportion by accompaniment model, by regimen, and at each time point ( 1 week after taking the first dose, 3 weeks after taking the first dose and study end). Additional subgroup analyses will include effectiveness by gestational age $(<7$ weeks' (up to 48 days), 7-9 weeks' (49-63 days) and 9+ through 12 weeks' (64-84 days) gestation); effectiveness by number of misoprostol doses for misoprostol-only regimens (comparing up to three doses vs four or more doses); effectiveness by number of attempts for combined mifepristone and misoprostol regimens (comparing first attempt: one tablet of mifepristone followed by up to three doses of misoprostol vs the second or any subsequent attempt: defined as a process that begins with an additional dose of mifepristone). We will also assess overall effectiveness, defined as the proportion of participants who report a complete abortion by the end of follow-up, inclusive of participants that reported a surgical intervention.

To assess a secondary outcome, whether self-managed medication abortion with accompaniment group support is non-inferior to medication abortion administered in clinical settings, we will calculate a pseudo-experimental non-inferiority test. We refer to this as a "pseudoexperimental' non-inferiority test, rather than a standard 


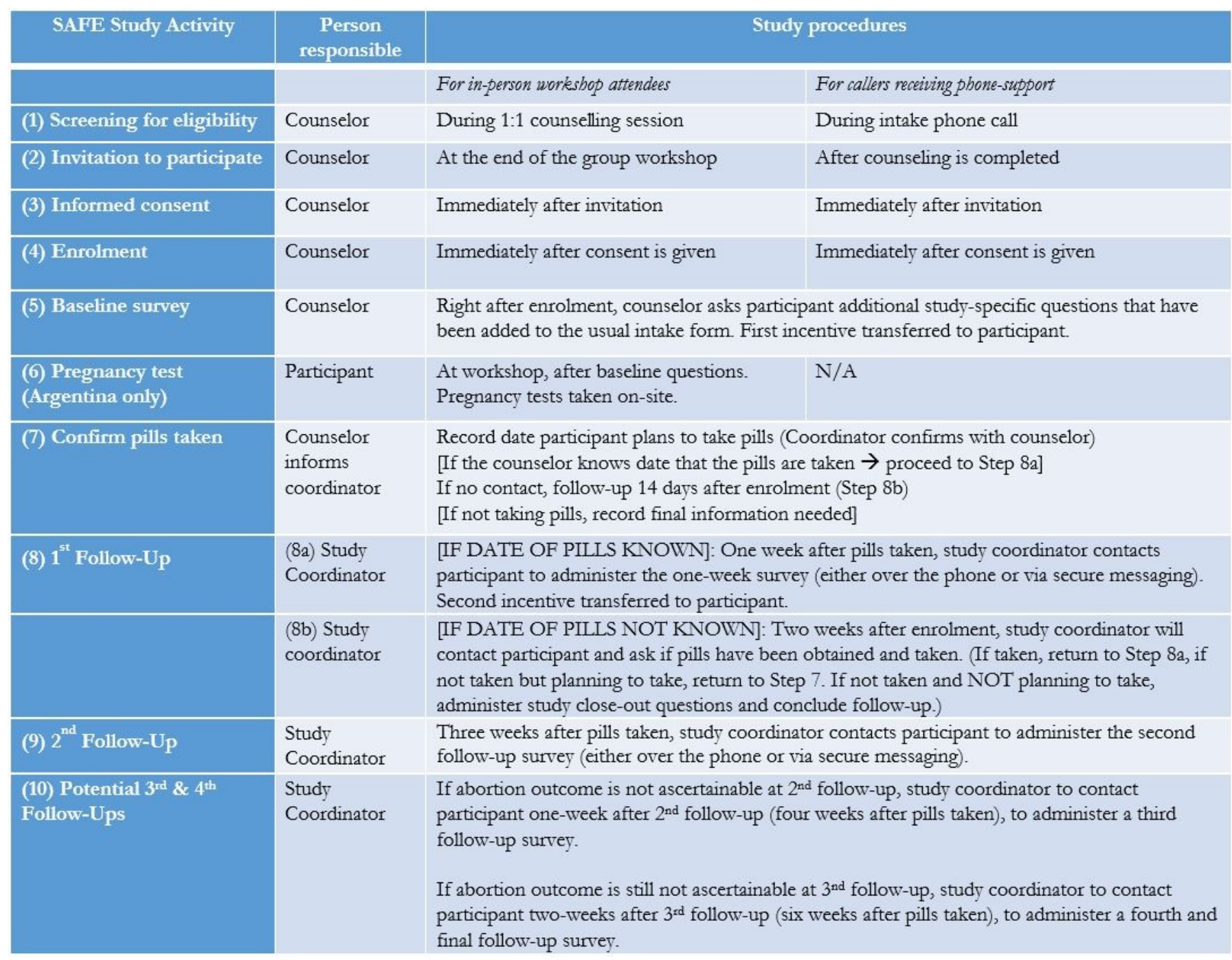

Figure 1 SAFE Study procedures. N/A, not available; SAFE Study, Studying Accompaniment model Feasibility and Effectiveness Study.

non-inferiority test, because we will compare the proportion who have a complete abortion within our prospective observational study to the proportion who had a complete abortion in a non-concurrent, historical control from randomised clinical trials. This is non-traditional, and pseudo-experimental, because treatment was not randomised in our observational study, and we are not comparing to a concurrent control arm wherein treatment was randomised. For this pseudo-experimental noninferiority test, we will calculate the difference $(D)$ in the proportion of those with complete abortions in the study sample $\left(p_{T}\right)$, as compared with the proportion of participants with complete abortions in historical controls $\left(p_{c}\right)$, and assess whether the difference is less than or equal to a prespecified margin of interest $(\delta) .{ }^{33-35}$ Data for the historical controls will be pulled from randomised clinical trials selected to most closely match the two medication abortion regimens endorsed by the accompaniment groups in the SAFE Study. ${ }^{28}{ }^{36-38}$ Similar to the primary effectiveness outcome, non-inferiority analyses will include only those participants who took the medications and have a known abortion outcome. Additionally, we will match participants on gestational age to comparison clinical trial data. The below equations specify the null and alternative hypotheses:

$$
\begin{aligned}
& H_{0}: D=p_{C}-p_{T} \geq \delta \\
& H_{A}: D=p_{C}-p_{T}<\delta
\end{aligned}
$$

The null hypothesis $\left(\mathrm{H}_{0}\right)$ states that the proportion with complete abortions in the SAFE Study $\left(p_{T}\right)$ is inferior to the proportion with complete abortions in the comparison clinical studies $\left(p_{C}\right)$. The alternative hypothesis $\left(\mathrm{H}_{\mathrm{A}}\right)$ states that the proportion with complete abortions in the SAFE Study $\left(p_{T}\right)$ is non-inferior to the proportion with complete abortions in the comparison clinical studies $\left(p_{C}\right){ }^{34}$ To test for non-inferiority, we will compute a onesided $95 \%$ CI for the difference in proportions $\left(p_{C}-p_{T}\right)$. The one-sided upper confidence bound for the difference is given by:

$$
U B=\hat{p}_{C}-\hat{p}_{T}+\sqrt[z_{1}-\alpha]{\frac{\hat{p}_{T}\left(1-\hat{p}_{T}\right)}{n_{T}}+\frac{\hat{p}_{C}\left(1-\hat{p}_{C}\right)}{n_{C}}}
$$


where $\hat{p}_{C}$ and $\hat{p}_{T}$ are the observed proportions of success in the SAFE Study and the clinical standard (93\% for mifepristone and misoprostol regimens, $80 \%$ for misoprostol-only regimens), respectively; $n_{C}$ and $n_{T}$ are the sample sizes of the corresponding groups, and $z_{1-\alpha}$ is the $(1-\alpha)$-percentile of a standard normal distribution. ${ }^{33-35}$ We will reject the null hypothesis if $\mathrm{UB} \leq \delta$.

We hypothesise that the effectiveness of self-managed medication abortion with accompaniment group support is non-inferior to the effectiveness of medication abortion administered in a clinical setting within a $5 \%$ margin of interest $(\delta=0.05)$. A $5 \%$ difference in effectiveness is small enough as to be well within the variation in medication abortion effectiveness measured in clinical studies and meets assumptions with regard to the superiority of the control regimen to placebo, thereby meeting core clinical and statistical considerations in the determination of the margin of interest $(\delta) .^{34}$

Additional secondary outcomes will include descriptive analyses of signs of complication, time to expulsion, ongoing pregnancy and medical treatment/surgical intervention. All outcomes will be measured by participant self-report at each follow-up. These secondary outcomes are defined below:

- Signs of complication: the proportion of participants who report experiencing heavy bleeding, extreme pain, foul-smelling discharge or high fever at any point in follow-up.

- Time to expulsion: range of time (in hours) from time from first medication dose to expulsion; as well as the proportion that experienced expulsion relative to medication dose (expulsion after the first, second, third or additional doses) as reported at last recorded follow-up. Kaplan-Meier methods will be used to model time to expulsion by regimen, stratified by gestational age.

- Ongoing pregnancy: proportion of participants who report an ongoing pregnancy at last recorded study follow-up.

- Medical treatment/surgical intervention: the proportion of participants who report receiving medical treatment and/or surgical intervention (actual receipt of medical care, beyond observation) at any point in follow-up.

We will report results by medication regimen (mifepristone and misoprostol in combination vs misoprostol alone); and pooled across regimens. Analysis of intraclass correlation coefficients for completion outcomes by counsellor in the pilot study suggests no correlation in outcomes within counsellors. ${ }^{27}$ All analyses will be conducted using the Stata and/or R statistical software programs, and results will be reported in accordance with the Strengthening the Reporting of Observational Studies in Epidemiology guidelines.

\section{Sensitivity/bias analyses}

We plan to conduct probabilistic multiple bias analyses to model the effectiveness of self-managed medication abortion under different combinations of misclassification, loss to follow-up and inclusion scenarios. The necessary bias parameters are: (a) sensitivity and specificity of self-report of abortion completion, (b) inclusion probabilities of all eligible clients based on abortion completion status and (c) loss to follow-up probabilities based on abortion completion status. These bias parameters will be estimated using appropriate probability distributions. Inclusion probabilities and lost to follow-up probabilities will be estimated using supplementary anonymised data from all clients who received medication abortion information from the organisations during the study period. Sensitivity and specificity of participant self-report of abortion completion will be estimated based on estimates from the literature, as well as compared with completion assessed by negative pregnancy test, reported ultrasound results, descriptions of products of conception and other abortion experiences assessed in follow-up.

\section{ETHICS AND DISSEMINATION}

There are several relevant ethical concerns for this study. First and foremost, there is a risk that if non-study personnel with negative intentions somehow access study data and are able to ascertain participant identity, that individual participants could face legal repercussions and/or negative social pressure. However, we will not collect any personally identifying information beyond contact information, and rigorous data security protocols minimise the risk of a data breach. Further, we will not collect data that would place participants at any additional risk beyond the data that the organisations are already collecting as part of their standard of care.

An additional potential risk is that participants might feel uncomfortable when responding to certain questions; however, participants are reminded that they can skip any question they do not wish to answer, and can withdraw from the study with no consequences for the counselling services they receive from the accompaniment group. Further, data collectors will be trained to guide participants to find safe and confidential spaces where they can speak (or message) freely, without fear of discovery.

We have been authorised to obtain verbal consent from participants. As the most serious risk associated with participation in the study is the potential loss of confidentiality, and because names and signatures on a consent form would be clearly identifiable information, we will substantially reduce this risk by not obtaining written consent. Rather, the accompaniment counsellor that enrols the participant will sign a consent form to indicate that the person has granted their verbal consent.

As the study eligibility criteria allow for any participant aged 13 years and up, it is possible that minors will participate in the study. These accompaniment services are accessible to and used by minors without parental consent. Requesting parental consent from minors to take part in this study could signify a potential risk to them, as obtaining consent to participate in the study would 
result in unwanted disclosure of pregnancy and abortion seeking. Within the countries in which the proposed research will be conducted, a waiver of parental consent can be granted when the proposed study (a) poses no more than minimal risk; (b) holds potential to benefit the minors being involved in the study and (c) the study objectives could not otherwise be achieved if parents were consented. Minors are an important group to include in research as little is known about the experiences of minors in self-managed abortion. Given the potential additional risks if parental consent is required, and the right of minors to assent to accompaniment services, we will use the same consent/assent form for all participants of the study regardless of their age. We do not foresee any additional risk to minors who choose to participate in this study.

This study protocol has been approved by the Allendale Investigational Review Board - the institutional review board (IRB) of record for the study - in March of 2019 and amended in July of 2019. In Argentina, the Fundación Huésped IRB approved the country-specific protocol. On requests from local implementing partners in Nigeria, the Allendale Investigational Review Board served as the IRB of record for the study. The protocol has also been submitted and reviewed by the study-specific DMOC, comprised of researchers with expertise in reproductive health research who reside in and/or are from Argentina, Nigeria and Southeast Asia.

The findings from this multinational study will inform the global conversation around the de-medicalisation of abortion services in both legally permissive and restrictive settings. Results will provide detailed information on the effectiveness and safety of self-managed medication abortion, and insight into if and how effectiveness and safety vary depending on other aspects of the experience. Findings related to the physical experience of abortion (onset and duration of bleeding, cramping, pain) can be used to better counsel and prepare people considering self-managed medication abortion. These findings can also help people better identify which physical symptoms are a cause for concern versus those that are a normal part of the medication abortion process. Findings related to people's experiences seeking care within the formal health system, including how they described their situation, the care they received and more will provide important insights into how to ensure that people are able to access medical care when they need it, without fear of mistreatment or legal prosecution.

Findings from the SAFE Study will be widely disseminated to researchers, advocates, healthcare providers, and stakeholders in non-clinical and clinical abortion provision alike to contribute to the evidence base on the effectiveness and safety of alternative models of medication abortion provision. In scientific settings, study results will be published in peer-reviewed journals in the global health field and submitted as scientific abstracts to relevant conferences. Beyond these scientific avenues of dissemination, we will also work closely with our partners and members of our research consortium to design a lay dissemination strategy that is appropriate and widereaching, including interactive web-based and paperbased briefs that highlight key findings; press releases to ensure that key findings from the study are shared with the public more broadly; and the development of blog posts, short video segments, and/or web graphics or other formats deemed appropriate by our partners that can be shared widely in digital format, via social media or other means, across multiple countries.

A definitive evaluation of the safety and effectiveness of self-managed medication abortion with accompaniment support could be instrumental in encouraging researchers, clinicians, advocates, and policymakers to revise and update current guidelines on the use of abortifacient medications outside of the formal health system. If found to be effective and safe, liberalisation of medication abortion guidelines could dramatically expand access to medication abortion, with enormous implications for reductions in morbidity and mortality due to unsafe abortion, and revolutionary implications for the human right to bodily autonomy.

\section{Author affiliations}

${ }^{1}$ Ibis Reproductive Health, Oakland, California, USA

${ }^{2}$ Ibis Reproductive Health, Cambridge, Massachusetts, USA

${ }^{3}$ Rollins School of Public Health, Emory University, Atlanta, Georgia, USA

${ }^{4}$ bis Reproductive Health, Johannesburg, Gauteng, South Africa

${ }^{5}$ Generation Initiative for Women and Youth Nigeria, Lagos, Nigeria

${ }^{6}$ Colectiva Feminista La Revuelta, Neuquen, Argentina

${ }^{7}$ Samsara, Yogyakarta, Indonesia

${ }^{8}$ Icahn School of Medicine at Mount Sinai, New York, New York, USA

Acknowledgements We wish to thank llana Dzuba, Onikepe Owolabi and Mariana Romero, who contributed to this work.

Contributors BK-0, CG, HM and RTJ conceived the initial study idea and obtained funding for the research. All authors (BK-0, CG, HM, RTJ, RM, CB, IE, BG, IAK, SN, RZ and EF) then collaborated to finalise the study conceptualisation, and to determine details and practicalities of study design and procedures that formed this study protocol. HM, BK-0, SF and RTJ drafted the initial study protocol, and all authors (BK-0, CG, HM, RTJ, RM, CB, IE, BG, IAK, SN, RZ and EF) then critically reviewed, edited and approved the final version to be published. All authors agree to be accountable for all aspects of the submitted work.

Funding A grant from the David and Lucille Packard Foundation funds the Studying Accompaniment model Feasibility and Effectiveness (SAFE) Study.

Competing interests None declared.

Patient consent for publication Not required.

Provenance and peer review Not commissioned; externally peer reviewed.

Supplemental material This content has been supplied by the author(s). It has not been vetted by BMJ Publishing Group Limited (BMJ) and may not have been peer-reviewed. Any opinions or recommendations discussed are solely those of the author(s) and are not endorsed by BMJ. BMJ disclaims all liability and responsibility arising from any reliance placed on the content. Where the content includes any translated material, BMJ does not warrant the accuracy and reliability of the translations (including but not limited to local regulations, clinical guidelines, terminology, drug names and drug dosages), and is not responsible for any error and/or omissions arising from translation and adaptation or otherwise.

Open access This is an open access article distributed in accordance with the Creative Commons Attribution Non Commercial (CC BY-NC 4.0) license, which permits others to distribute, remix, adapt, build upon this work non-commercially, and license their derivative works on different terms, provided the original work is properly cited, appropriate credit is given, any changes made indicated, and the use is non-commercial. See: http://creativecommons.org/licenses/by-nc/4.0/. 
ORCID iD

Heidi Moseson http://orcid.org/0000-0002-2488-2429

\section{REFERENCES}

1 Baum S, DePiñeres T, Grossman D. Delays and barriers to care in Colombia among women obtaining legal first- and second-trimester abortion. Int J Gynaecol Obstet 2015;131:285-8.

2 Finer LB, Frohwirth LF, Dauphinee LA, et al. Timing of steps and reasons for delays in obtaining abortions in the United States. Contraception 2006;74:334-44.

3 Gerdts C, Fuentes L, Grossman D, et al. Impact of clinic closures on women obtaining abortion services after implementation of a restrictive law in Texas. Am J Public Health 2016;106:857-64.

4 Kiley JW, Yee LM, Niemi CM, et al. Delays in request for pregnancy termination: comparison of patients in the first and second trimesters. Contraception 2010;81:446-51.

5 Aiken ARA, Digol I, Trussell J, et al. Self reported outcomes and adverse events after medical abortion through online telemedicine: population based study in the Republic of Ireland and Northern Ireland. BMJ 2017;357:j2011.

6 Endler M, Beets L, Gemzell Danielsson K, et al. Safety and acceptability of medical abortion through telemedicine after 9 weeks of gestation: a population-based cohort study. BJOG 2019;126:609-18.

7 Footman K, Keenan K, Reiss K, et al. Medical abortion provision by pharmacies and drug sellers in low- and middle-income countries: a systematic review. Stud Fam Plann 2018;49:57-70.

8 Foster AM, Arnott G, Hobstetter M. Community-Based distribution of misoprostol for early abortion: evaluation of a program along the Thailand-Burma border. Contraception 2017;96:242-7.

9 Moseson H, Herold S, Filippa S, et al. Self-managed abortion: a systematic scoping review. Best Pract Res Clin Obstet Gynaecol 2020;63:87-110.

10 Gomperts RJ, Jelinska K, Davies S, et al. Using telemedicine for termination of pregnancy with mifepristone and misoprostol in settings where there is no access to safe services. BJOG 2008;115:1171-8.

11 Ganatra B, Gerdts C, Rossier C, et al. Global, regional, and subregional classification of abortions by safety, 2010-14: estimates from a Bayesian hierarchical model. Lancet 2017;390:2372-81.

12 Singh S, Shekhar C, Acharya R, et al. The incidence of abortion and unintended pregnancy in India, 2015. Lancet Glob Health 2018;6:e111-20.

13 Singer EO. Realizing abortion rights at the margins of legality in Mexico. Med Anthropol 2019;38:167-81.

14 Zurbriggen R, Keefe-Oates B, Gerdts C. Accompaniment of secondtrimester abortions: the model of the feminist Socorrista network of Argentina. Contraception 2018;97:108-15.

15 World Health Organization (WHO) DoRHaR. Health worker roles in providing safe abortion care and post-abortion contraception. Geneva, Switzerland: World Health Organization, 2015.

16 Jain JK, Dutton C, Harwood B, et al. A prospective randomized, double-blinded, placebo-controlled trial comparing mifepristone and vaginal misoprostol to vaginal misoprostol alone for elective termination of early pregnancy. Hum Reprod 2002;17:1477-82.

17 Chen MJ, Creinin MD. Mifepristone with buccal misoprostol for medical abortion: a systematic review. Obstet Gynecol 2015;126:12-21.

18 Ngoc NTN, Blum J, Raghavan S, et al. Comparing two early medical abortion regimens: mifepristone+misoprostol vs. misoprostol alone. Contraception 2011;83:410-7.
19 Ashok PW, Templeton A, Wagaarachchi PT, et al. Factors affecting the outcome of early medical abortion: a review of 4132 consecutive cases. BJOG 2002;109:1281-9.

20 Dalenda C, Ines N, Fathia B, et al. Two medical abortion regimens for late first-trimester termination of pregnancy: a prospective randomized trial. Contraception 2010;81:323-7.

21 Kapp N, Grossman D, Jackson E, et al. A research agenda for moving early medical pregnancy termination over the counter. BJOG 2017;124:1646-52.

22 Gerdts C, Jayaweera RT, Baum SE, et al. Second-Trimester medication abortion outside the clinic setting: an analysis of electronic client records from a safe abortion Hotline in Indonesia. BMJ Sex Reprod Health 2018:286-91.

23 Gomperts R, van der Vleuten K, Jelinska K, et al. Provision of medical abortion using telemedicine in Brazil. Contraception 2014;89:129-33.

24 CRR, The World's Abortion Laws. The definitive record of the legal status of abortion in countries across the globe, updated in real time, 2019. Available: https://reproductiverights.org/worldabortionlaws [Accessed 5 Dec 2019].

25 WHO. Medical management of abortion. Geneva, Switzerland: World Health Organization, 2018.

26 Blumenthal PD, Clark S, Coyaji KJ, et al. Providing medical abortion in low-resource settings: an introductory guidebook. 2nd ed. New York: Gynuity Health Projects, 2009.

27 Moseson H, Jayaweera R, Raifman S, et al. Self-managed medication abortion outcomes: results from a prospective pilot study. Reprod Health 2020;17:164.

28 von Hertzen H, Piaggio G, Huong NTM, et al. Efficacy of two intervals and two routes of administration of misoprostol for termination of early pregnancy: a randomised controlled equivalence trial. Lancet 2007;369:1938-46.

29 Hamoda H, Ashok PW, Flett GMM, et al. A randomized trial of mifepristone in combination with misoprostol administered sublingually or vaginally for medical abortion at 13-20 weeks gestation. Hum Reprod 2005;20:2348-54.

30 Winikoff B, Dzuba IG, Chong E, et al. Extending outpatient medical abortion services through 70 days of gestational age. Obstet Gynecol 2012;120:1070-6.

31 ANSIRH. Analysis of Medication Abortion Risk and the FDA report "Mifepristone U.S. Post-Marketing Adverse Events Summary through 12/31/2018". San Francisco, CA, USA: Advancing New Standards in Reproductive Health (ANSIRH) University of California, 2019.

32 Schmidt-Hansen M, Cameron S, Lohr PA, et al. Follow-up strategies to confirm the success of medical abortion of pregnancies up to 10 weeks' gestation: a systematic review with meta-analyses. Am J Obstet Gynecol 2020;222:551-63.

33 Tunes da Silva G, Logan BR, Klein JP. Methods for equivalence and noninferiority testing. Biol Blood Marrow Transplant 2009;15:120-7.

34 D'Agostino RB, Massaro JM, Sullivan LM. Non-inferiority trials: design concepts and issues - the encounters of academic consultants in statistics. Stat Med 2003;22:169-86.

35 Phillips KF. A new test of non-inferiority for anti-infective trials. Stat Med 2003;22:201-12.

36 Tang OS, Xu J, Cheng L, et al. Pilot study on the use of sublingual misoprostol with mifepristone in termination of first trimester pregnancy up to 9 weeks gestation. Hum Reprod 2002;17:1738-40.

37 Tang OS, Chan CCW, Ng EHY, et al. A prospective, randomized, placebo-controlled trial on the use of mifepristone with sublingual or vaginal misoprostol for medical abortions of less than 9 weeks gestation. Hum Reprod 2003;18:2315-8.

38 von Hertzen H, Huong NTM, Piaggio G, et al. Misoprostol dose and route after mifepristone for early medical abortion: a randomised controlled noninferiority trial. BJOG 2010;117:1186-96. 